\title{
HTCC based Ku/IF/BB Down Converter for satellite on board processing applications
}

\author{
M.C.Comparini*, C.Leone*, P.Montanucci*, M.Tursini** \\ *Alenia Spazio S.p.A., Equipment Engineering Unit \\ Via Saccomuro 24, 00131 Roma, Italy \\ **Alenia Spazio S.p.A., Microelectronic Technology Unit \\ V. Pile 60, 67100 L'Aquila, Italy
}

Phone: +390641512488Ｆax: +390641512507Ｅmail: c.leone@roma.alespazio.it

\begin{abstract}
This paper presents the demonstration of an ultra compact High Temperature Cofired Ceramic (HTCC) based down converter for satellite on board processing equipment. The down converter is composed of three sections: the RF Front end in $\mathrm{KU}$ Band, the first IF at $400 \mathrm{MHz}$ and a base-band chain at $50 \mathrm{MHz}$. The overall gain is of $90 \mathrm{~dB}$ and the OIP3 is of $20 \mathrm{dBm}$. GaAs MMICs, Si RF-IC and ceramic saw filter have been integrated on a compact HTCC module of a size of $59 \times 66 \times 6 \mathrm{~mm}$.
\end{abstract}

\section{INTRODUCTION}

Global communication is dramatically changing the life of people. Internet services explosion has demonstrated how interactivity is today a real powerful instrument: users located in all over the world need to access to information in all its forms and communication networks are evolving to meet increasing demands for higher speed and wider bandwidth. A satellite based solution has already demonstrated its appeal to create new service opportunities: using Ka band opens the door to multigigabit systems, providing point to multi-point and point to point communications among fixed terminals for business application as intranet and videoconferencing, fast internet access for both business and residential users, and mobile or transportable terminals for private user applications, such as the Internet, file transfer, tele-education and tele-working (1). The key feature is the use of on-board regeneration and base band switching.

\section{ELECTRICAL DESIGN AND PERFORMANCES}

The down converter receives the signals transmitted in MF-TDMA format from the KA band receiver and provides a suitable signal to the digital demodulation section.

The design goals has been the optimisation of the unit key performances such as mass, power consumption and dimensions and a tuning-less approach (2).

The electrical and technological design must address in fact not only the electrical performance issue but also the production aspects considering the high number of functions integrated in the payload. The processing capacity of each demodulator module, which is composed of a down converter function and a digital section, is about $18 \mathrm{Mbps}$, whose format is in accordance with the up-link signal structures. It means that for each gigabit traffic the number of down converters and demodulators is more than fifty. The keys to achieve such results are the use of advanced technologies for better performances and reliability of the equipment.

The down converter RF section is composed of a low noise amplifier, an hybrid balanced mixer on alumina substrate which converts the RF signal down to IF frequency, providing strong rejection of LO harmonics and a multiplied by two on LO2 path (3). The two times multiplier is a custom PHEMT MMIC, realized by a European GaAs foundry (Figure 11). The IF chain provides the channelisation. The main characteristics of the IF amplifiers are low noise figure, high gain and high output third intercept point. Low noise figure and high gain have to be provided to guarantee the noise figure of the overall Front End because of the Saw Filter high insertion loss. The Saw Filter provides an efficient utilisation of the spectrum and high selectivity in small size: the device has an ultimate rejection of about $40 \mathrm{~dB}$ which allows to reduce the adjacent channel interference level.

The base-band chain is composed of a custom RF IC Analog ASIC (Figure 12) integrating the mixer and amplification functions (4), a low pass filter for cutting all the mixer leakage signals that might be present at the input of the amplifiers. The amplifier inside the Analog Asic provides also the AGC (Automatic Gain Control) function (control range $45 \mathrm{~dB}$ ). A Medium Power Amplifier (MPA) is used as the output stage in order to have a good linearity at high output levels. 
The block schematic is shown in Figure 1.

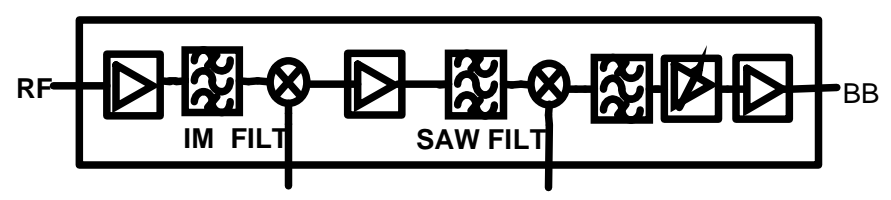

Figure 1: DownConverter Block Schematic

\section{HTCC MODULE TECHNOLOGY}

The Down Converter is a MHIC (Microwave Hybrid Integrated Circuit) realized with ISP (Integrated Substrate Package) manufactured by NTK onto an Alenia Spazio design (Figure 2). All three RF sections $(\mathrm{Ku}, \mathrm{IF}$ and $\mathrm{BB})$ are integrated in a unique, small size, HTCC multilayer substrate. RF striplines, microstrips, DC supply and control circuitry lines are integrated in the multilayer substrate. Electrical isolation between supply and RF lines in the ISP ceramic substrate is done by internal ground planes. $\mathrm{Ku}$ band mixer, band pass filter and striplines have been manufactured with alumina substrates glued on ISP, metallized with thin film processes to reach 30 micron line/gap resolution. Physical and electrical separation between $\mathrm{Ku}$, IF and $\mathrm{BB}$ section is obtained with internal walls of a metallic frame, hermetically brazed onto the HTCC substrate. A metallic cover, laser welded on brazed frame top side, guarantee the ISP hybrid hermetic closure and electrical shielding of RF sections. EMI and radiation shielding is obtained with a metallic, not hermetic box (the Down Converter unit package) onto which ISP hybrid, DC and SMA connectors are mounted.

Input signals can reach the internal part of ISP hybrid through ceramic transitions hermetically brazed between the substrate and the frame. Transition band is from 0 to $20 \mathrm{GHz}$.

HTCC substrate material is AlN, a ceramic with a thermal conductivity one order greater than standard alumina substrates $\left(140\right.$ vs $\left.16 \mathrm{~W} / \mathrm{m}^{\circ} \mathrm{C}\right)$. This choice was caused by the need to reduce the operating temperature of some GaAs small size amplifiers, which have an high power dissipation density $(350 \mathrm{~mW}$ in $0.83 \times 0.83 \mathrm{~mm}^{2}$ area). AlN ceramic permit to reach a chip backside operating temperature calculated value of $89{ }^{\circ} \mathrm{C}$ (mounting plate temp. : $60^{\circ} \mathrm{C}$ ), while alumina substrates would have implied $140^{\circ} \mathrm{C}$ (max allowable $\left.\mathrm{Tj}=105^{\circ} \mathrm{C}\right)$.

Box material is an AlSi alloy with high silicon percentage, a lightweight and thermally conductive material whose thermal expansion coefficient is significantly close to the HTCC substrate one, in order to guarantee a reliable bonding between the ISP and the box.

The use of bare Si and GaAs component, of a ceramic SAW filter, of an ISP package with all lines and interconnections buried in the substrate, has permitted an high integration of the down converter unit, with significant volume (ISP: 59x66x6 mm.; whole unit : $75 \times 71 \times 14 \mathrm{~mm}$.) and weight (ISP: 44 grams; whole unit about 130 grams) reduction.

Manufacturing process consist now only in the construction of a single hybrid circuit, instead of many circuits pre-assy and test, and a final assy of the different circuits in the unit that must be finally tested, so production phase is simplified and manufacturing lead time is significantly reduced (50\%). Due to the ISP small size, automatic die attach and wire bonding can be easily performed, to further reduce manufacturing lead time and cost.

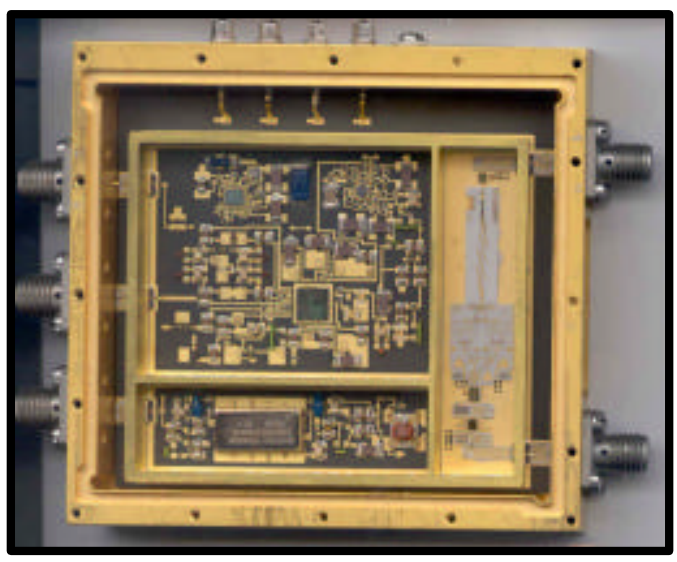

Figure 2: DownConverter photo

The HTCC module is composed of four layers: on the top layer all the GaAs and Silicon active components and all passives are mounted, alumina substrates are glued on the gold plated RF section, from I and Q ports of the image rejection mixer two striplines are designed to obtain the same electrical lenght on the inner layer2, to be compliant of the image rejection frequency specification. The inner layer1 is continuous ground plane, gone through via holes bringing the power supply and control signal. The bottom layer is continuous ground. The ground planes are connected together through via holes and the five wrap-around ground underneath the RF input and output transitions.

Table 1

Material Characteristics

\begin{tabular}{|c|c|}
\hline & ALN \\
\hline Conductor & W \\
\hline $\begin{array}{c}\text { Dielectric constant } \\
\text { (at 10GHz) }\end{array}$ & 8.5 \\
\hline $\begin{array}{c}\text { Dielectric loss } \\
\text { (at 10GHz) }\end{array}$ & 0.003 \\
\hline $\begin{array}{c}\text { T.C.E. } \\
\text { [ppm/deg.C] }\end{array}$ & 4.4 \\
\hline $\begin{array}{c}\text { Thermal Conductivity } \\
\text { [W/mK.R.T.] }\end{array}$ & 200 \\
\hline $\begin{array}{c}\text { Flexural strength } \\
\text { [Mpa] }\end{array}$ & 170 \\
\hline
\end{tabular}




\section{EXPERIMENTAL RESULTS}

In the following Figures (3-10) HTCC Downconverter measurements plots are shown.

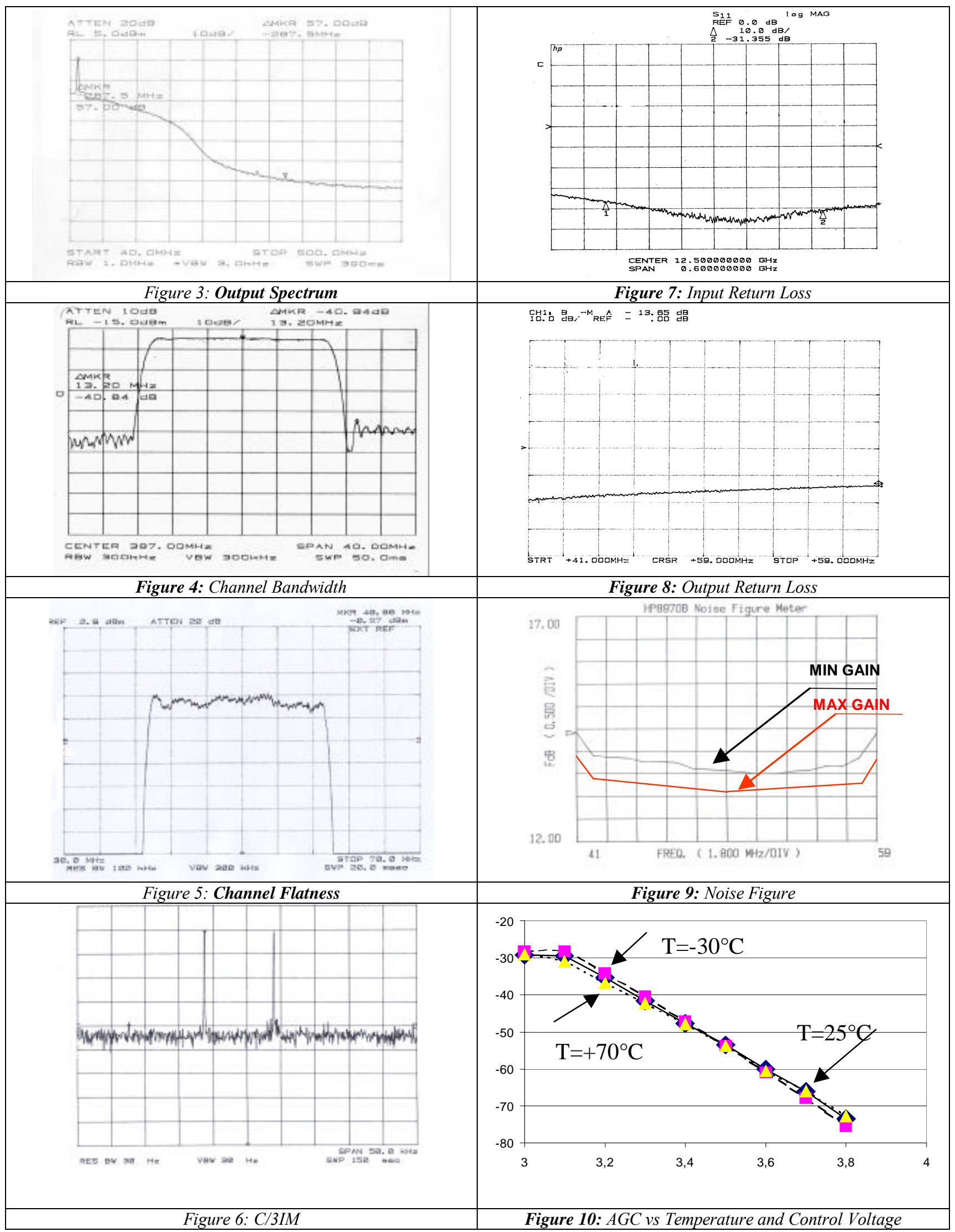


Table 2

DWC Measurements

\begin{tabular}{|c|c|}
\hline Parameter & \\
\hline Input Frequency & $11-14 \mathrm{GHz}$ \\
\hline Noise Figure & $15 \mathrm{~dB}$ \\
\hline Max Gain & $90 \mathrm{~dB}$ \\
\hline C/3IM @ input level=-90dBm & $40 \mathrm{dBc}$ \\
\hline AGC Control Range & $45 \mathrm{~dB}$ \\
\hline Return Loss & $15 \mathrm{~dB}$ \\
\hline Weight & $130 \mathrm{~g}$ \\
\hline
\end{tabular}

\section{FUTURE WORKS}

On the basis of the results of the RF Section of the Demodulator, the next step is the integration between the Ku/IF/BB DownConverter with the Digital Section, for testing the up-link signal. Both sections are in ceramic multi-layers substrate, the digital one is in LTCC (Low Temperature Cofired Ceramic), and it has the same dimension of the RF section to be finally integrated in the same housing.

\section{CONCLUSION}

The use of advanced technologies in packaging, High Temperature Cofiring Ceramic multilayer substrate, die attach, wire bonding and the use of GaAs MMICs and RF-IC for Radio Frequency electrical design have allowed an improvement of electrical performances due to the good isolation between RF and DC path, very low weight, good thermal conductivity and low manufacturing lead time. A laser welding process is used to hermetically sealed covers. It has proven a very reproducible, fast process guaranteeing high reliability and repeatable hermeticity; excellent for high volume production.

\section{ACKNOWLEDGEMENT}

The authors would like to thank NTK staff for his support, in particular Mr. H. Sanchez and Mr. N. Miyawaki

\section{REFERENCES}

(1) M.Marinelli, R.Giubilei "A regenerative payload for satellite multimedia communication" IEEE Multimedia, Dec. 99

(2) M.C.Comparini "Microwave Technology for space application: a fast evolution to be competitive"European Microwave Week 2000, Paris, October 2000

(3) M.C.Comparini, C.Leone et al. "Next Generation Payload Equipment For Broadband Multimedia Satellite Communication" European Microwave Week 2001, London, September 2001

(4) F.Adirosi M.C.Comparini, C.Leone "Application of silicon based RF IC devices in space communication systems \& equipment"-European Microwave Week 2000, Paris, October 2000

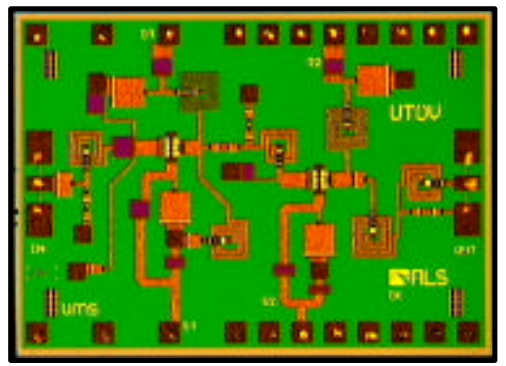

Figure 11: MMIC X2 Multiplier

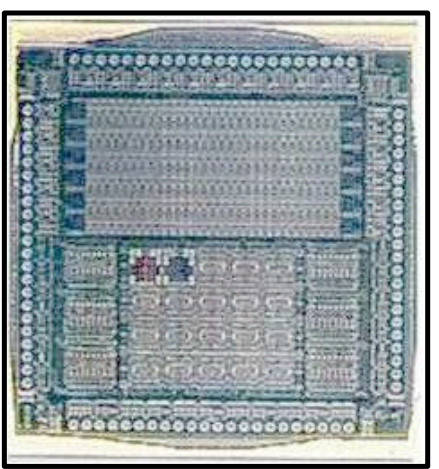

Figure 12: RFIC Si IF DownConverter 